\title{
Subgrid particle method for porous media and suspension flow
}

\author{
R. G. M. van der Sman \& G. Brans \\ Food and Bioprocess Engineering, Wageningen University, \\ The Netherlands
}

\begin{abstract}
In this contribution we report on the initial steps in the development of a numerical scheme for flow through packed or suspended spheres. The spheres are semiresolved, meaning that their diameter is smaller than the grid spacing, but their excluded volume is taken into account. Flow in the fluid phase is solved using the volume-averaged equations. Particle motion is solved via Newtons law, taking into account drag force and lubrication forces only. Despite the low resolution of the flow field, the particle trajectories of two spheres colliding in shear flow can be reasonable reproduced.

Keywords: Lattice Boltzmann, porous media, suspension flow, Euler-Lagrangian.
\end{abstract}

\section{Subgrid particle method}

The subgrid particle method is a Euler-Lagrangian method for modeling the multiphase flow problem of gas fluidised beds, cf. [1-3]. Here particles are underresolved, meaning that their radius is smaller than the grid spacing, but their volume is excluded for the fluid.

In this paper we present an extension of the subgrid particle method towards suspension flow, requiring that hydrodynamic interactions mediated via the liquid has to be included. Such a scheme for suspensions has been proposed by Schwarzer [4], but has been implemented in 2D. The model presented in this paper is 3D, and is implemented in Lattice Boltzmann. Lattice Boltzmann is chosen as it has shown to be a very versatile method capable of simulating a variety of complex fluids. Next to suspensions and fluidized beds, the subgrid particle method can also be applied to porous media. Governing equations for porous media flow and fluidized bed are identical if solid phase is assumed immobile. 
The validity of the method is shown via the simulation of several benchmark problems. We investigate the Beaver-Joseph problem of flow in a channel partially filled with a porous layer [5]. This problem is challenging due to the stepwise change in porosity, and poses for many numerical schemes serious problems $[6,12]$. For the subgrid particle method for suspension flow it is important to handle the problems with changing porosity, i.e. solid fraction. Subsequently, we investigate the classical problem of two particle colliding in shear flow, as first investigated by Batchelor and Green [7]. This is a good problem for testing the validity of hydrodynamic interaction.

\section{Governing equations for fluid phase}

For the governing equations for the fluid phase we follow those of Feng and $\mathrm{Yu}$ [1].

$$
\begin{aligned}
\partial_{t} \rho \phi & =\partial_{\alpha} \rho \phi u_{\alpha} \\
\partial_{t} \rho \phi u_{\alpha}+\partial_{\beta} \rho \phi u_{\alpha} u_{\beta} & =-\partial_{\alpha} p+\partial_{\beta} \phi \mu\left(\partial_{\beta} u_{\alpha}+\partial_{\alpha} u_{\beta}\right)+F_{d r a g, \alpha}
\end{aligned}
$$

Here $\rho$ is the bulk density of the fluid, $\phi$ is the porosity, $u_{\alpha}$ is the component of the velocity field in the Cartesian direction $x_{\alpha} . p$ is the pressure, and $\mu$ is the dynamic viscosity of the fluid. We assume that particles are neutrally buoyant. We note that the superficial velocity field is $\tilde{u}_{\alpha}=\phi u_{\alpha}$. In suspension flow $F_{d r a g, \alpha}$ is the backflow induced by the drag of the fluid on the particles. Below we explain how this is computed in case of suspension flow. For porous media flow it follows from closure relations, such as the Ergun relations. In case of negligible inertia the above equation is equal to the Darcy-Forchheimer-Brinkman equation. The Ergun relation for a randomly packed bed of spheres in the Stokes flow regime is:

$$
F_{d r a g, \alpha}=\frac{180 \mu(1-\phi)^{2}}{\phi^{2} d_{p}^{2}} u_{\alpha}
$$

\section{LB scheme for flow through inhomogeneous porous media}

With the Lattice Boltzmann scheme [8] we simulate the above governing equations for the fluid phase. Via inverse Chapman-Enskog expansion we have derived this LB scheme. In order to model spatially varying porosity stability of the scheme requires that the speed of sound should be less than the regular value: $c_{s}^{2}<c^{2} / 3$, This induces however anisotropy in the viscosity $\mu$ [9]. This can be remediated via a modified MRT scheme [10]. We briefly present the resulting LB scheme. More details will be presented in a full length paper. In LB physical fields are represented by moments of particle distribution function $f_{i}$. These particles move over a simple cubic Bravais lattice with lattice spacing $\Delta x$ with discrete set of velocities $c_{i, \alpha}=\Delta x_{i, \alpha} / \Delta t$ which brings them to nearest and next-nearest neighbouring lattice sites. Upon arrival the particles collide with each other, and after collision they 
propagate with their new velocity. The collide and propagation steps are described by a discrete version of the Boltzmann equation:

$$
f_{i}\left(\mathbf{x}+\Delta x_{i}, t+\Delta t\right)-f_{i}(\mathbf{x}, t)=-\Omega_{i j} f_{j}^{n e q}(\mathbf{x}, t)
$$

with $f_{i}^{n e q}=f_{i}-f_{i}^{e q}$ the non-equilibrium part of the distribution function. The moments equilibrium part of the distribution function $f_{i}^{e q}$ determines the governing physics, as shown by the inverse Chapman-Enskog expansion [9]. The collison rate is controlled by the scattering matrix $\Omega_{i j}$. In the commonly used Lattice BGK scheme $\Omega_{i j}$ is linear with the unit matrix. But here we require the multiple relaxation scheme (MRT), which has a scattering matrix with different eigenvalues.

The moments of the equilibrium distribution, as follows from the inverse Chapman-Enskog expansion, are equal to:

$$
\begin{gathered}
M_{0}^{e q}=\sum_{i} f_{i}^{e q}=\phi \rho \\
M_{1, \alpha}^{e q}=\sum_{i} f_{i}^{e q} c_{i, \alpha}=\phi \rho u_{\alpha} \\
M_{2, \alpha \beta}^{e q}=\sum_{i} f_{i}^{e q} c_{i, \alpha} c_{i, \beta}=\rho c_{s}^{2} \delta_{\alpha \beta}+\phi \rho u_{\alpha} u_{\beta} \\
M_{3, \alpha \beta \gamma}^{e q}=\sum_{i} f_{i}^{e q} c_{i, \alpha} c_{i, \beta} c_{i, \gamma}=\rho c_{s}^{2}\left(u_{\alpha} \delta_{\beta \gamma}+u_{\beta} \delta_{\alpha \gamma}+u_{\gamma} \delta_{\alpha \beta}\right)
\end{gathered}
$$

Note that $M_{0}^{e q}$ and $M_{1, \alpha}^{e q}$ are conserved quantities: density and momentum.

In the MRT scheme before collision the particle distribution function is projected on the set of eigenvector, which happen to be Hermite tensor polynomials [9]. Each moment of the distribution function is relaxed towards its equilibrium value, at a rate determined by its associated eigenvalue. After collision the moments are projected back to the particle distribution function, and are propagated to the adjacent lattice sites.

For the implementation of the drag body force, we have implemented the scheme of Ladd, who proposed to apply one half of the forcing before the collision step, and the other half after the collision.

\subsection{Numerical tests}

The above presented LB scheme is validated using the benchmark problem of flow in a flat channel partly filled with a porous layer. The porous layer has a height $H$, and the clear fluid layer has a height $L_{y}$. An analytical solution to this problem is by Beavers and Joseph [5]. Beavers and Joseph have treated the problem as a two domain problem, with different governing equations. Hence, at the interface of the two domains the boundary conditions have to be matched via a closure relations. Goyeau and coworkers have proposed a single domain approach, with one governing equation, namely Eq.(1) [12]. At the interface between fluid and porous medium they assumed continuity of the superficial velocity and the stress. 


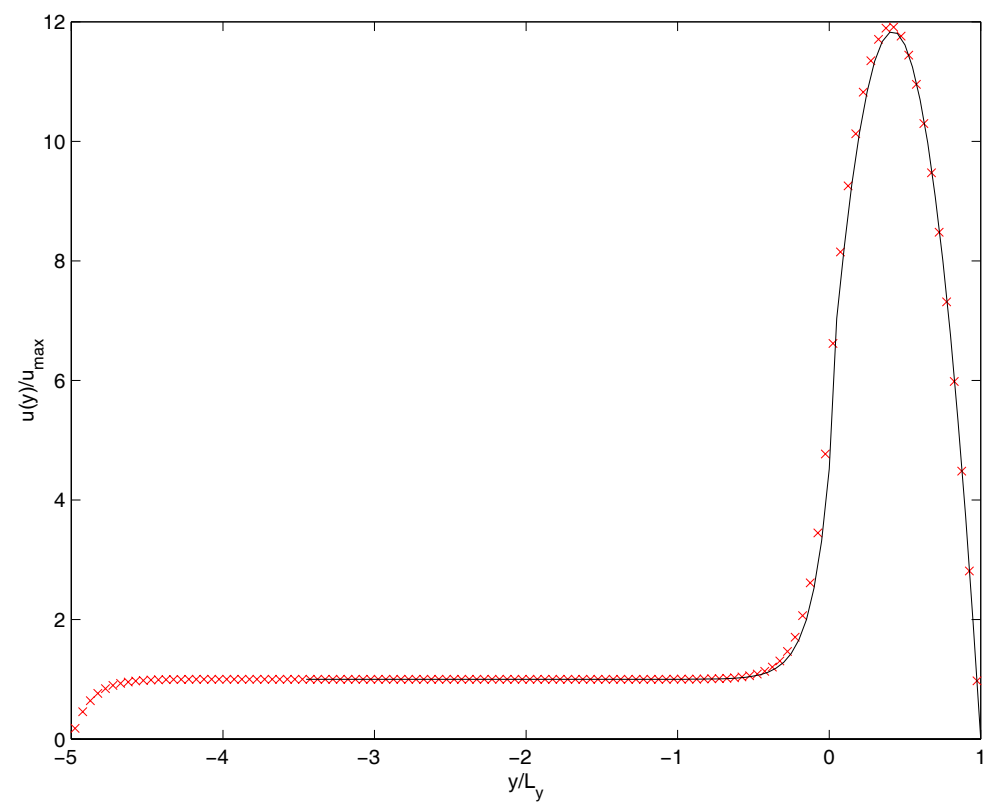

Figure 1: Analytical and numerical solution of flow in porous layer adjacent to fluid in a channel between parallel plates, with $D a=0.08$, and $\phi_{0}=0.8$.

Their numerical solution agrees with the analytical solution of Beavers and Joseph, if a gradual change of porosity at the interface is assumed.

We have checked the results of Goyeau and coworkers. We have varied porosity as $\phi=\phi_{0}+\frac{1}{2}(1-\tanh (y / \zeta))\left(1-\phi_{0}\right)$, with $\phi_{0}$ the porosity of the porous layer. Simulations are performed with a $120 \times 10 \times 1$ lattice, $\phi_{0}=0.8$, Darcy number $D a=0.08, \zeta / \Delta x=1$, porous layer height $H / L_{y}=6$, and Reynolds number $R e=u_{\text {max }} L_{y} / v=0.7$. Results are shown in figure 1 , from which we observe that our model shows good agreement with the analytical solution.

\section{Suspension flow}

\subsection{Lagrangian scheme}

In this section we describe how the solid phase in suspension flow is treated in our scheme. Subsequently, we test the validity of this part using the benchmark problem of Green and Batchelor.

Subgrid particles have diameter $d_{p} \ll \Delta x_{i}$ and mass $m_{p}$. We solve their motion via Newtons law:

$$
m_{p} \frac{d \mathbf{v}_{p}}{d t}=m_{p} \mathbf{a}_{p}=\mathbf{F}_{d r a g, p}+\mathbf{F}_{p q}+\mathbf{F}_{\text {wall }, p}
$$


with $F_{d r a g, p}$ the drag force on the particle $p, F_{p q}$ the force due to hydrodynamic interaction between particles $p$ and $q$. Between particles and confining wall there is also hydrodynamic interaction. For other applications Newtons law is easily extended with gravity or Brownian forces.

The drag force on the particle follows the correlation of van der Hoef [13]:

$$
\mathbf{F}_{\text {drag }, p}=\frac{G(\phi)}{\phi} 3 \pi \mu d_{p}\left(\mathbf{u}-\mathbf{v}_{p}\right)
$$

with the factor

$$
G(\phi)=\frac{10(1-\phi)}{\phi^{2}}+\phi^{2}(1+1.5 \sqrt{1-\phi})
$$

Note that in the limit of dilute suspensions, $G(\phi) \rightarrow 1$, and the drag force is equal to the Stokes drag force.

The drag force is coupled back to the fluid as backflow, following Newtons third law. If the boundaries of lattice cells intersect the volume of the particle, the force will be distributed over the lattice cells intersecting the particle, proportional to the volume fraction of the particle in the particular lattice cell, cf. [3]. In similar way, the solid volume fraction is distributed over the lattice cells. In this way the porosity field $\phi$ is computed.

The hydrodynamic interaction between particles with same diameter is given by the two-point lubrication force. As in regular Lattice Boltzmann part of the hydrodynamic interaction is already resolved. Hence, we take a modified lubrication force:

$$
F_{p q, \alpha}=-6 \pi \mu d_{p}^{2}\left(v_{p, \alpha}-v_{q, \alpha}\right) \hat{e}_{r, \alpha}\left(\frac{1}{h}-\frac{1}{h_{c}}\right)
$$

Note that $r$ is the distance between the particles, which all have the same diameter $d_{p}$. The gap between particles is $h=r-d_{p}$. The lubrication force is proportional to the relative velocity between the particles. $h_{c}$ is a cut off length where the lubrication force is zero.

If the gap between particles is smaller than $h_{\min }$ a spring force acts between particles $p$ and $q$ :

$$
F_{\text {spring, } \alpha}=-k\left(h-h_{\min }\right) \hat{e}_{r, \alpha}
$$

The wall lubrication force acts only if the gap is within the cut off distance $h<h_{c}$ :

$$
\mathbf{F}_{\text {wall }, l u b}=-6 \pi d_{p}^{2} \mu v_{p, n}\left(\frac{1}{h}-\frac{1}{h_{c}}\right) \hat{e}_{n}
$$

Here $\hat{e}_{n}$ is the outward pointing normal vector to the confining plane. If the gap is even smaller $h<h_{\min }$ the spring force is acting on the particle, similar as above. 
As common in molecular dynamics Newtons law is integrated via a velocityVerlet scheme:

$$
\begin{aligned}
& r_{p, \alpha}(t+\delta t)=r_{p, \alpha}(t)+v_{p, \alpha} \delta t+\frac{1}{2} a_{p, \alpha} \delta t^{2} \\
& v_{p, \alpha}(t+\delta t)=v_{p, \alpha}(t)+a_{p, \alpha} \delta t
\end{aligned}
$$

The timestep $\delta t$ can vary during simulation, and will be determined by the smallest gap between particles, as shown below. Stability of the scheme set some requirements to the time scales involved. Below we list the time scales 1) convective time scale $\left.\tau_{\phi}=d_{p} / v_{p}, 2\right)$ time scale of drag force: $\left.\tau_{\beta}=\rho_{p} d_{p}^{2} / 18 \rho_{f} v, 3\right)$ kinematic time $\left.\tau_{v}=d_{p}^{2} / \nu, 4\right)$ spring force time scale: $\tau_{k}^{2}=k_{\text {spring }} / m_{p}$ 5) lubrication force time scale $\tau_{l u b}=\rho_{p} d_{p} h^{\prime} / 36 \rho_{f} v$. with $1 / h^{\prime}=1 / h-1 / h_{c}$. Note that dimensionless numbers can be expressed in ratios of the above time scales, namely the Reynolds number: $R e=\tau_{\nu} / \tau_{\phi}$, and the Stokes number $S t=\tau_{\zeta} / \tau_{\phi}$. Stability sets the following hierarchy of time scales: $\delta t \ll \tau_{l u b}<\tau_{\zeta}<\tau_{v} \ll \tau_{\phi}$.

\subsection{Numerical test}

We compare the collision of two equal spheres in linear shear field with the solution of Batchelor and Green. Results are shown in figure 2. Results are obtain
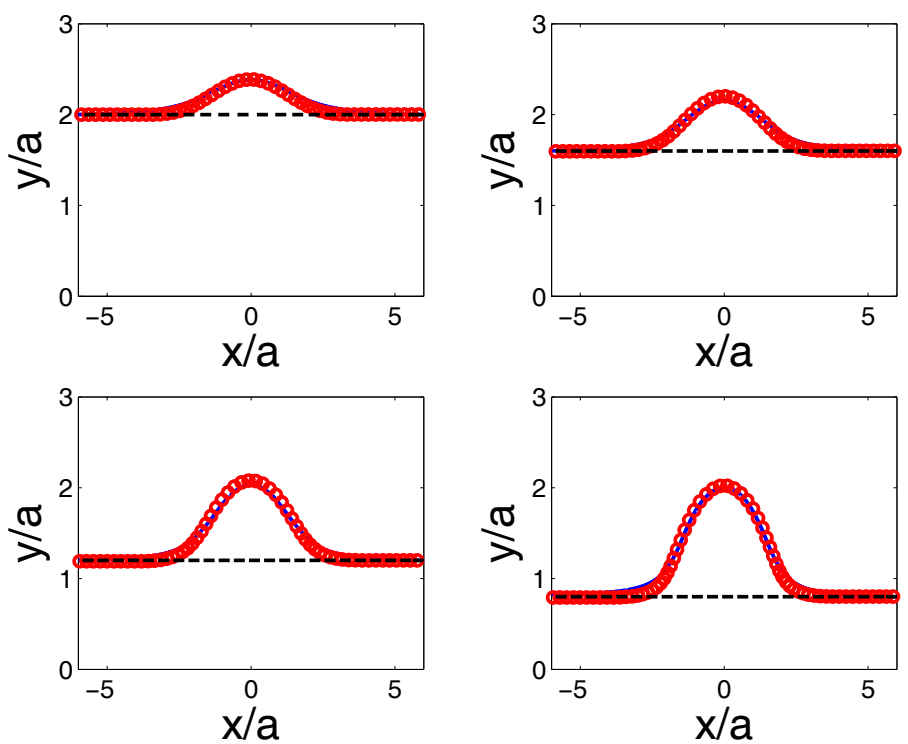

Figure 2: Normalised relative trajectory of a sphere colliding with a second sphere in a linear shear field. Solid lines indicate the solution given by Batchelor and Green, and symbols indicate our numerical solution. Dashed line indicates the base line to which the sphere should return after collision. 


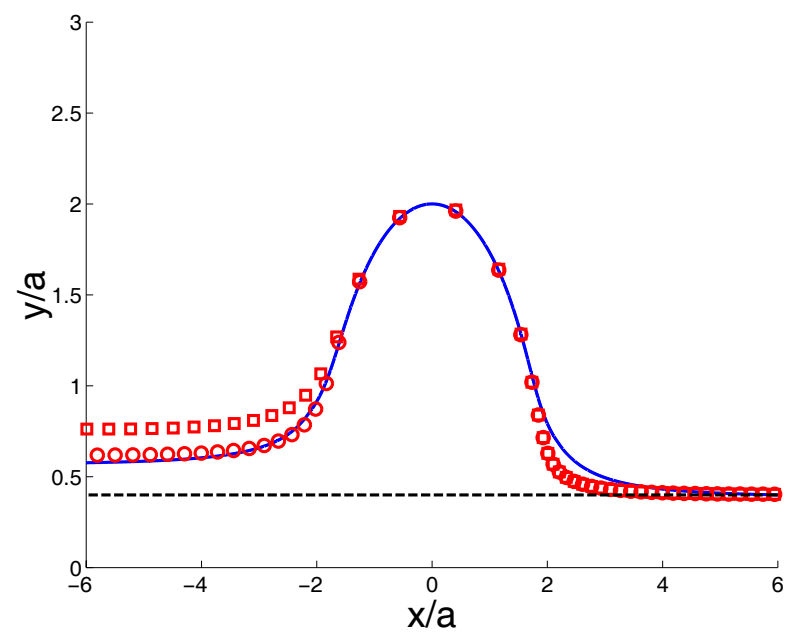

Figure 3: Normalised relative trajectory of a sphere colliding with a second sphere in a linear shear field, with initial vertical distance $y / a=0.4$ and $h_{\min }=$ $0.01 a$, and $h_{\min }=0.004 a$.

with cut off length for the lubrication $h_{c}=2 d_{p}, d_{p}=0.2 \Delta x$. Initial positions of the particles are at $x_{0}= \pm 5 d_{p}$, and $y_{0}= \pm \frac{1}{2 m} d_{p}$ with $m=\{1,2, \ldots, 5\}$. In figure 2 we show the particle trajectory (with respect to the barycentre) for $m=2, \ldots, 5$. Here we have set $h_{\min }=0.01 d_{p}$. Surprisingly we obtain very good agreement with the analytical solution of Batchelor and Green, although the flow is not fully resolved. In figure 3 we show results for $m=1$, where the particle come very close in contact. In this case we have changed $h_{\min }$ to $h_{\min }=0.005 d_{p}$ and $h_{\min }=0.002 d_{p}$. We observe that the particles do not return to their initial streamlines. Still some asymmetry in the analytical solution. Might be due to the fact that tangential lubrication is not included [14].

This error improves if we take smaller $h_{\mathrm{min}}$, at the cost of a significant increase of computing time, as smaller gaps lead to smaller subgrid time steps $\delta t$. At $h_{\min }=$ $0.005 d_{p}$ we obtain nearly the same solution as the integration of the analytical solution of the particle velocities given by Batchelor and Green.

\section{Conclusions}

In this paper we have given a first introduction to a numerical scheme describing flow through porous media and suspensions at a semi-resolved scale. We have obtained reasonable agreement with benchmark problems. Currently we are investigating problems of suspensions with a multitude of particles. 


\section{Acknowledgements}

We thanks Prof. John Brady, Prof. Wim Briels, Prof. Mike Cates and dr. Kevin Stratford for useful discussions. The stay of Gerben Brans at the group of prof. Cates, Univ. Edinburgh is financially supported by HPC-Europe programme.

\section{References}

[1] Y.Q. Feng and A.B. Yu. Assessment of model formulations in the discrete particle simulation of gas-solid flow. Ind. Chem. Res. 43: 8378-8390 (2004).

[2] Y.P. Tsuo and D. Gidaspow. Computation of flow pattern in circulating fluidized beds. AIChE J. 36(6): 885-896 (1990).

[3] B.P.B. Hoomans, J.A.M. Kuipers, W.J. Briels, and W.P.M. van Swaaij. Discrete particle simulation of bubble and slug formation in a two dimensional gas-fluidised bed: a hard sphere approach. Chem Eng. Sci. 51: 99-118 (1996).

[4] S. Schwarzer. Sedimentation and flow through porous media: simulating dynamically coupled discrete and continuum phases. Phys. Rev. E 52(6): 6461-6475 (1995).

[5] G.S. Beavers, and D.D. Joseph. Boundary conditions at a naturally permeable wall. J. Fluid Mech. 30: 197-207 (1967).

[6] J.A. Ochoa-Tapia, and S. Whitaker. Momentum-transfer at the boundary between a porous medium and a homogenous fluid .1. theoretical development. Int. J. Heat Mass Transfer 38(14): 2635-2646 (1995).

[7] G.K. Batchelor, and J.T. Green. Hydrodynamic interaction of 2 small freelymoving spheres in a linear flow field. J. Fluid Mech. 56: 375-400 (1972).

[8] S. Chen, and G.D. Doolen. Lattice Boltzmann method for fluid flows Ann. Rev. Fluid Mech. 30: 329-364 (1998).

[9] R.G.M. van der Sman Galilean invariant lattice Boltzmann scheme for natural convection on square and rectangular lattices. Phys. Rev. E 74(2): 026705 (2006).

[10] R.G.M. van der Sman, and S. Bogale. Porous media and suspension flow with modified MRT Lattice Boltzmann scheme. submitted to J. Comput. Phys. (2007).

[11] S. Whitaker. The Forchheimer equation: a theoretical development. Transp. Porous Media 25: 27-61 (1996).

[12] B. Goyeau, D. Lluillier, D. Gobin, and M.G. Velarde. Momentum transport at a fluid-porous interface. Int. J. Heat Mass transfer 46: 4071-4081 (2003).

[13] M.A. van der Hoef, R. Beetstra, and J.A.M. Kuipers. Lattice-Boltzmann simulations of low-Reynolds-number flow past mono- and bidisperse arrays of spheres: results for the permeability and drag force. J. Fluid Mech. 528: 233254 (2005).

[14] N.Q. Nguyen, and A.J.C. Ladd. Lubrication corrections for latticeBoltzmann simulations of particle suspensions. Phys. Rev. E 66(4): 046708 (2002). 